MATEC Web of Conferences 40, 02003 (2016)

DOI: $10.1051 /$ matecconf $/ 20164002003$

(c) Owned by the authors, published by EDP Sciences, 2016

\title{
CFD Analysis of The Hydraulic Turbine Draft Tube to Improve System Efficiency
}

\author{
Spandan Chakrabarty ${ }^{1}$, Bikash Kr. Sarkar ${ }^{2}$, Subhendu Maity ${ }^{2}$ \\ ${ }^{1}$ Siddaganga Institute of Technology, Tumkur, India \\ ${ }^{2}$ NIT Meghalaya, India
}

\begin{abstract}
Demand of the power is increasing day by day with the development of the science and technology. Development of the renewable energy sector has become essential issue at the present situation due to the limited source of the non-renewable energy. Hydro energy power generation sector is superior over the other renewable sector due to the high efficiency, ability to continuous generation and low generation cost. In India a great amount of the power generation is taken care by the hydro power system but still some more potential have unexplored. The efficiency improvement of the hydro turbine system can be done for the new installation or installed system by the improvement in component level. The system can be installed by the state of the art equipment, like modern inlet guide vane (IGV) control system, improved design of the runner, IGV system, draft tube, penstock to reduce the loss, hence improve the efficiency. The energy recovery in the draft tube depends on the design of draft tube. In the present work the optimized design of the draft tube shape through computational fluid dynamics (CFD) simulation has been carried out in ANSYS FLUENT platform. The design objective of the draft tube is to reduce the flow loss and improve the energy recovery, hence to improve the efficiency.
\end{abstract}

\section{Introduction}

Demand of the power is increasing day by day with the development of the science and technology. Development of the renewable energy sector has become essential issue at the present situation due to the limited source of the non-renewable energy. Hydro energy power generation sector is superior over the other renewable sector due to the high efficiency, ability to continuous generation and low generation cost. In India a great amount of the power generation is taken care by the hydro power system but still some more potential have unexplored. The efficiency improvement of the hydro turbine system can be done for the new installation or installed system by the improvement in component level. The system can be installed by the state of the art equipment, like modern inlet guide vane (IGV) control system, improved design of the runner, IGV system, draft tube, penstock to reduce the loss, hence improve the efficiency.

Hydraulic turbines are used to convert the energy of the flowing water to rotating mechanical energy, hence generate the electrical power. The main sources of the loss of the energy are the flow loss, loss in turbine IGV and loss of kinetic energy at the exit of the runner. The most of the scientist are focused on the turbine runner design for improve the system efficiency [1-3]. The draft tube is one of the important components of reaction hydraulic turbine is use to convert the dynamic pressure to static pressure recover the energy of the exit water from the turbine runner, which lead to increase the efficiency of the turbine. The draft tube is a divergent pipe connected at the exit of the runner to the tail race. The main functions of draft tube is to allow the installation of turbine above the tail race level without loss of head and to convert major part of kinetic energy at the runner outlet into pressure energy. The use of very low head and high speed turbines, the kinetic energy leaving the runner became higher and the height of the runner above the tail race became smaller. This is achieved increasing the cross-sectional area of the draft tube in the flow direction. Initially, the straight conical tubes with inlet and outlet areas of different cross section were used and part of kinetic energy could be converted into potential energy for use in the turbine. The straight draft tubes has been used in hydrodynamic investigations on 1903 and 1907 and investigators adopted walls of draft tube parallel to streamline based on theoretical solutions [4]. However, it did not find much practical application due to non-uniform distribution of flow at the entry, swirling of flow and vortex flow along the length of the tube. The straight tube draft tube restricted for small and medium size turbines. If the turbine runner is large the inlet diameter of the draft tube will be large, so the length of the draft tube is large. The large size draft tube for the turbine becomes irrational to construct. The bell mouth tube can be use for the recovery of the axial and the radial flow machines but the use of those types of draft tubes for large runner diameters has again restriction 
due to support problem due to the large dimensions and weight. All these problems can be resolved by the elbow tube draft tube. Recently the scientist have been considered soft computing technique [5] for the shape optimization of turbine draft tube.

In the present work the effect of the energy recovery on the shape of the draft tube has been studied by the computational fluid dynamics (CFD) approach in ANSYS FLUENT platform. To study the draft tube efficiency 3D viscous laminar flow has been considered. The velocity distribution and the pressure distribution throughout the draft tube has been studied, hence the draft tube efficiency has been estimated. The draft tube efficiency has been estimated for different geometry of the draft tube and optimum geometry has been found based on the maximum draft tube efficiency.

\section{Descriptions and modelling}

The geometry of the elbow draft tube has been considered for the flow analysis has been shown in the figure 1 and figure 2. Figure 1 shown the schematic view of the draft tube and the figure 2 shown the cad modelling of the draft tube has been built in ANSYS environment. The dimensions of the inlet outlet and the exit of the draft tube have been shown in the figure. The inlet of the draft tube has been considered as circular cross section the exit of the draft tube has been considered as the rectangular cross section. The draft tube consists of three main part like, conical section connected to the turbine runner, elbow section and the exit diffuser. To simplify the analysis and to keep geometric consistency $\alpha_{1}=\alpha_{2}$ and $\beta_{1}=\beta_{2}$ has been considered. The performance of the draft tube can be can be specified by the pressure recovery and the loss coefficient [5]. The average pressure recovery factor can be represent as,-

$$
C_{p}=\frac{\frac{1}{A_{3}} \int_{A_{3}} p d A-\frac{1}{A_{1}} \int_{A_{1}} p d A}{\frac{1}{2} \rho V_{1 a v}^{2}}
$$

Where $\boldsymbol{A}_{1}$ and $\boldsymbol{A}_{3}$ are the inlet and exit cross section area of the draft tube respectively, $V_{1 a v}$ is the average flow velocity of the inlet of the draft tube and $\rho$ is the density of the flowing water.

$$
A_{1}=\frac{\pi d_{1}^{2}}{4}
$$

$$
A_{3}=b_{2} \times h_{4}
$$

The flow loss coefficient can be represent as,-

$$
\varsigma=\frac{\frac{1}{A_{1}} \int_{A_{1}} p_{s t 1} \text { und } A-\frac{1}{A_{3}} \int_{A_{3}} p_{s t 3} \text { und } A}{\frac{1}{2} \rho V_{1 a v}^{2}}
$$

where $\mathrm{n}$ and $\mathrm{u}$ are the velocity vector and the surface normal vector respectively. $\boldsymbol{p}_{s t 1}$ and $\boldsymbol{p}_{s t 1}$ are the stagnation pressure at the inlet and outlet of the draft tube.
The draft tube efficiency can be represent as,

$$
\begin{gathered}
\eta=\frac{\Delta \boldsymbol{P}_{r}}{\boldsymbol{V}_{1}^{2}} \\
\Delta \boldsymbol{P}_{\boldsymbol{r}}=\left(\boldsymbol{P}_{3}-\boldsymbol{P}_{1}\right)-\boldsymbol{P}_{\boldsymbol{l}}
\end{gathered}
$$

Where $\eta_{\text {is the draft tube efficiency and the }} \Delta \boldsymbol{P}_{r}$ is the pressure recovery.

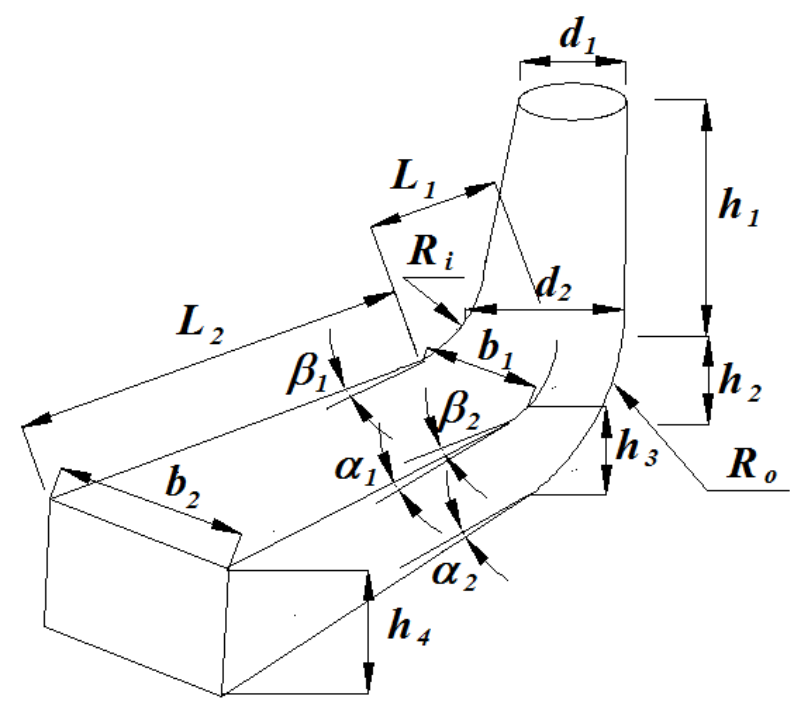

Figure1. Dimensions for the geometric modelling of the draft tube.

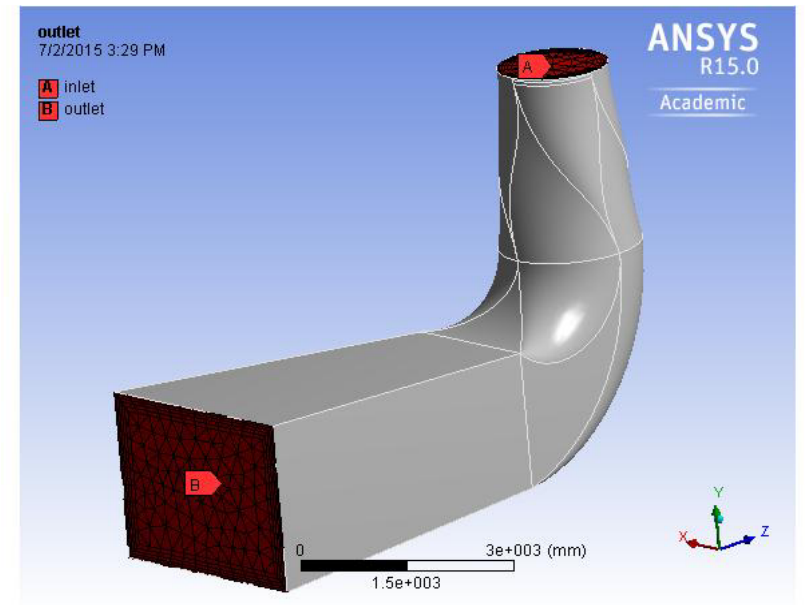

Figure2. Isometric view of the draft tube builds in ANSYS cad modelling.

At the exit of the Francis turbine pressure is the negative. The cone section has divergent section to increase the pressure and decrease the flow velocity. To take care of the pressure recovery in the draft tube diffuser tubes are chosen and hence the kinetic energy difference can be increased by either increasing diffuser angle of draft tube or its length. Corresponding to this there will be losses due to flow separation, swirling of flow and vortex flow along the length of draft tube and especially in curved type draft tubes there will be secondary flows and backpressure in flow after bend. Fluent analysis is made on various dimensions of the draft tubes and the optimum design of the draft tube is 
selected on the basis of maximum draft tube efficiency and to reduce the flow loss with respect to the draft tube geomatry.

\section{Simulation}

Three dimension laminar viscous flow field has been considered for the simulation of the hydraulic turbine draft tube design. The flow simulation has been performed based on the Nevier Stoke's equation consideration. To simulate the flow through the draft tube discretaization has been done by the tetrahedral meshing as shown in the figure 3 .

The initial geometric configuration parameter value of the large turbine draft tube has been considered for the simulation study with respect to the inlet diameter as $2.25 \mathrm{~m}$. The initial geometry of the draft tube has been shown in figure 2 and the corresponding meshing shown in figure 3.

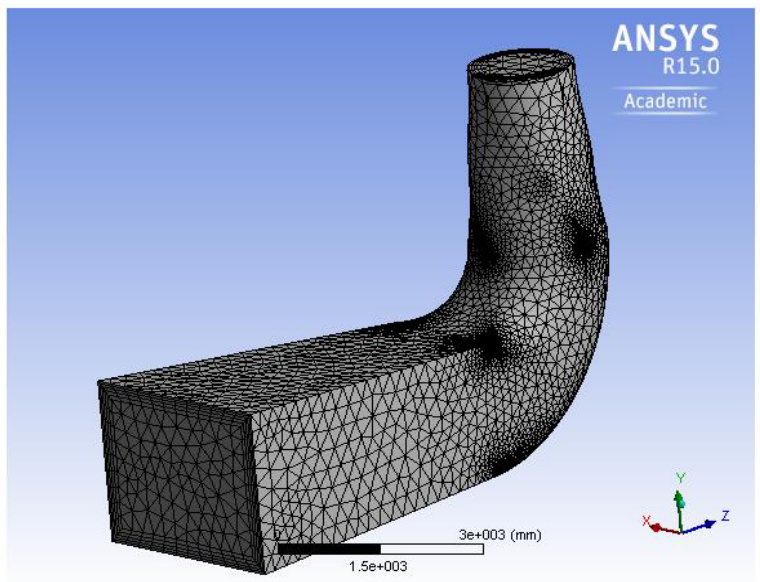

Figure3. Meshing of draft tube

The meshing of the draft tube flow domain is generated in ANSYS CFD MESH as shown in fig.3. The mesh generation involves specification of element length and to convert the large domain into number of small elements. Each element consists of nodes at which flow variables are calculated. The unstructured triangular elements on surfaces and tetrahedral in flow domain are adopted in draft tube meshing. The medium mesh is done near the surfaces in order to capture the boundary layer. A 5 layer boundary is specified at the inlet and outlet. This has reduced the number of grids points and hence computational time. The mass flow rate normal to surface at inlet of draft tube cone is specified as inlet boundary condition. The static pressure at outlet of draft tube is specified as outlet boundary condition. The walls of draft tubeare assumed to be smooth with no slip. Incompressible fluid, water is taken as the working fluid. ANSYS FLUENT is used as the solver for analysis boundary curvature in elbow and diffusing flow.
The steady state viscous 3D laminar flow analysis simulation has been carried out in elbow draft with initial inlet diameter $\boldsymbol{d}_{1}$ is $2.25 \mathrm{~m}$ and $\boldsymbol{h}_{1} / \boldsymbol{d}_{1} 1.304, \boldsymbol{b}_{1} / \boldsymbol{d}_{1}$ 1.111, $\boldsymbol{b}_{2} / \boldsymbol{d}_{1}$ is 2.11 and mass flow rate is of the water is 20000 $\mathrm{Kg} / \mathrm{s}$. The simulation result for this configuration has been shown in the figure 4, 5 and 6 . The result shown that at inlet of the draft tube flow velocity is high and the pressure is very low. As the flow comes down through the cone the flow velocity decreases and the pressure is increases. At the elbow section pressure is very high and at the exit the flow velocity is very small and it shows some tendency of the back flow.

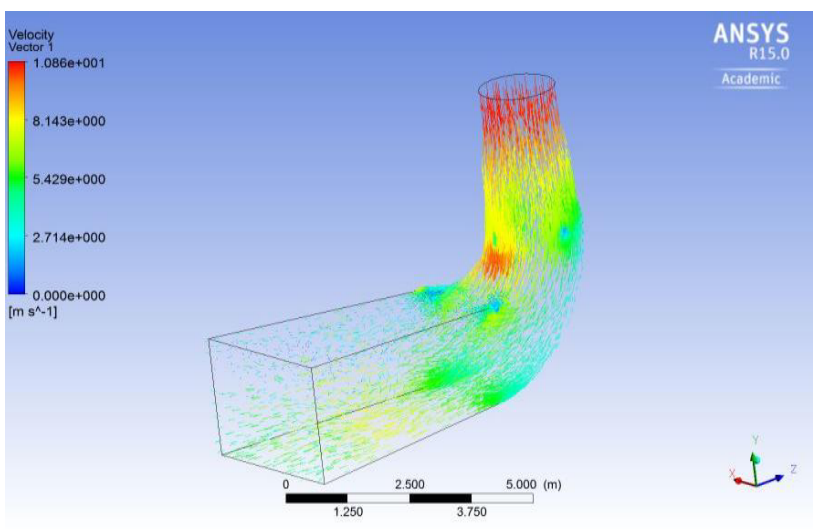

Figure 4. The velocity vector for draft tube geometry

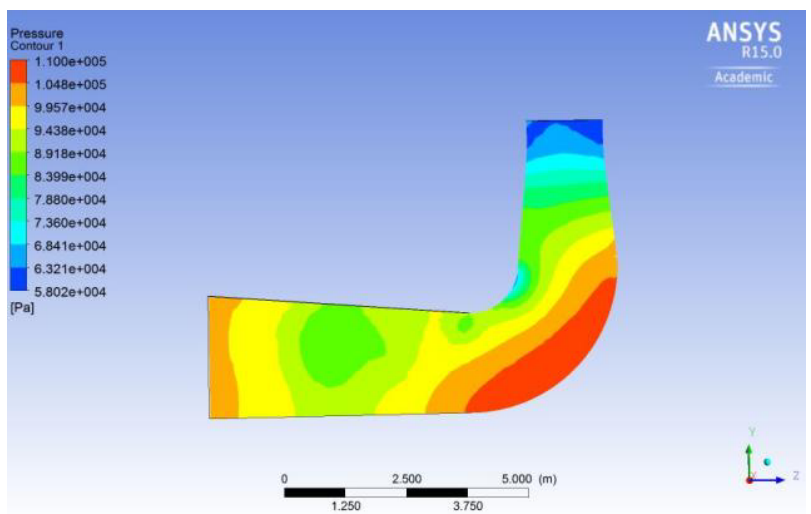

Figure 5. The pressure contour for draft tube geometry.

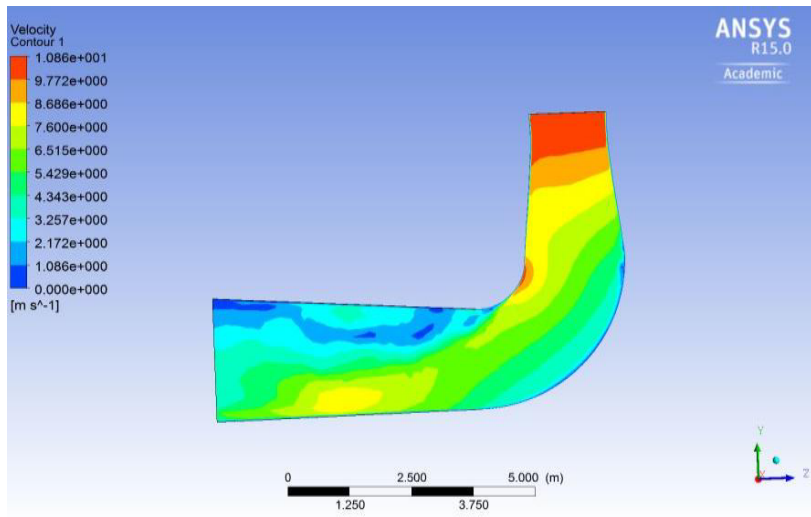

Figure 6. The velocity contour for draft tube geometry. 
The result from the simulation has been found as inlet velocity at the inlet of the draft tube is $10.86 \mathrm{~m} / \mathrm{s}$ and out let velocity as $2.172 \mathrm{~m} / \mathrm{s}$. The inlet pressure has been found as $58020 \mathrm{~Pa}$ and outlet as $104800 \mathrm{~Pa}$. The head loss comes out to be $1 \mathrm{~m}$ and the draft tube efficiency as $79.35 \%$. All the parameters value has been found from the equation (4), and (5).

The next geometry is taken for the simulation as $\boldsymbol{h}_{1} / \boldsymbol{d}_{1}$ is equal to $1.304, \boldsymbol{b}_{1} / \boldsymbol{d}_{1}$ as 1.222 and $\boldsymbol{b}_{2} / \boldsymbol{d}_{1}$ as 1.666 with a mass flow rate of $20000 \mathrm{~kg} / \mathrm{sec}$. FLUENT analysis is carried out and the velocity and pressure profiles are plotted as shown in figure 7 , figure 8 , and figure 9 .

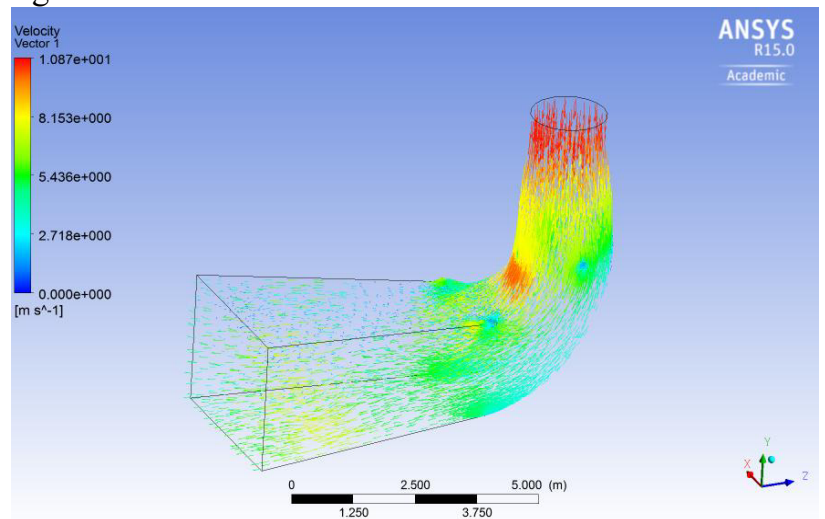

Figure 7. The velocity vector for draft tube geometry.

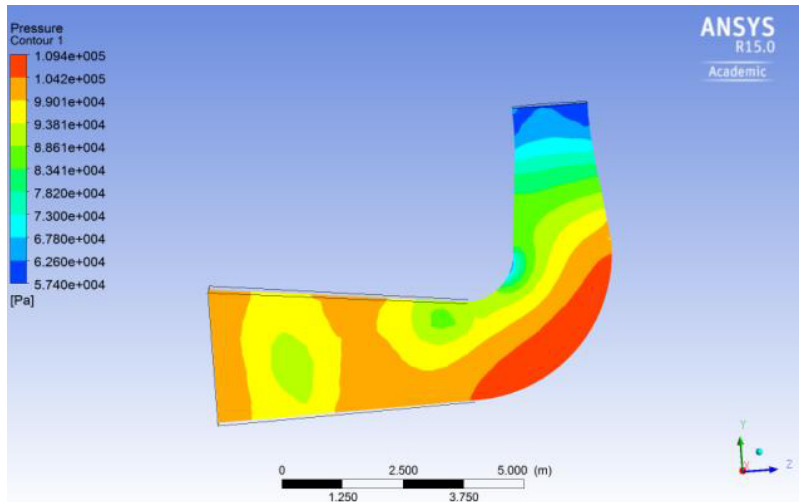

Figure 8. The pressure contour for draft tube geometry

The inlet estimated parameter value has been found as the velocity at the inlet is $10.87 \mathrm{~m} / \mathrm{s}$ and out let velocity as $2.174 \mathrm{~m} / \mathrm{s}$. The inlet pressure has been found as 57400 $\mathrm{Pa}$ and outlet as $104200 \mathrm{~Pa}$. The head loss comes out to be $1 \mathrm{~m}$ and the draft tube efficiency as $79.37 \%$. We notice that for a certain height increasing the outlet area has the slightest change in efficiency.

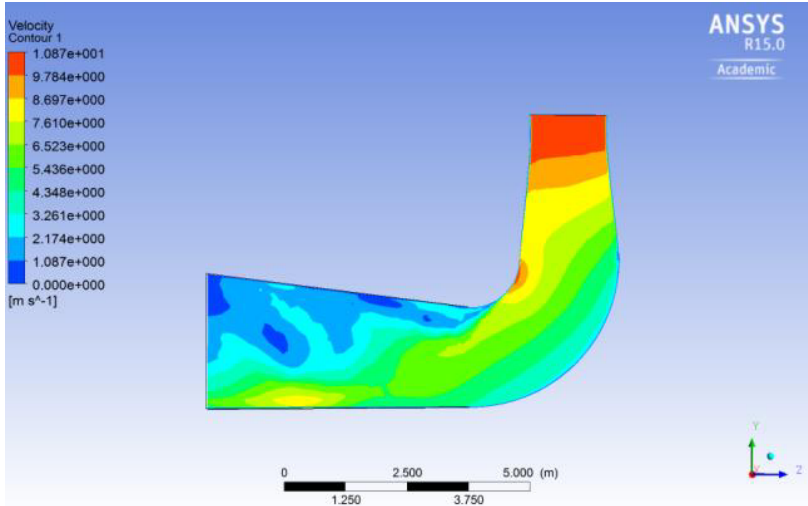

Figure 9. The velocity contour for draft tube geometry

The next geometry is taken for $\boldsymbol{h}_{1} / \boldsymbol{d}_{1}$ as 1.193, $\boldsymbol{b}_{1} / \boldsymbol{d}_{1}$ as 1.111 and $\boldsymbol{b}_{2} / \boldsymbol{d}_{1}$ as 1.444 with a mass flow rate of $20000 \mathrm{~kg} / \mathrm{sec}$, thus decreasing the outlet area. FLUENT analysis is carried out and the velocity and pressure profiles are plotted as shown in figure 10, figure 11 , and figure 12 .

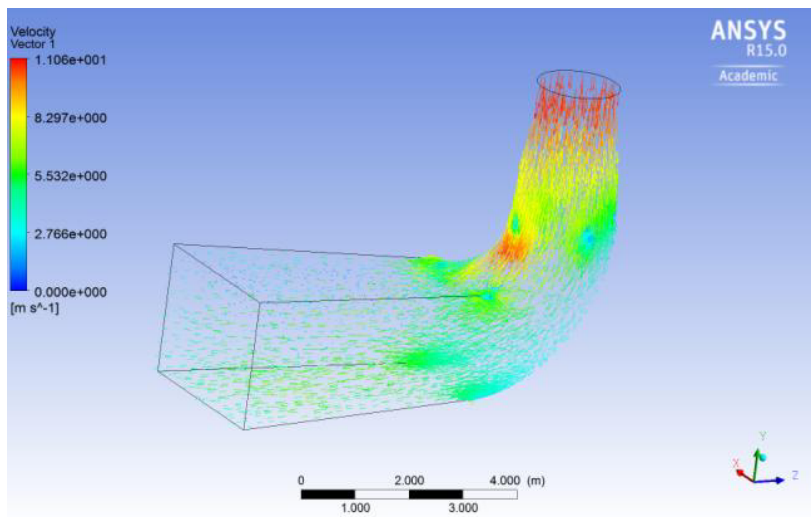

Figure10. The velocity vector for draft tube geometry.

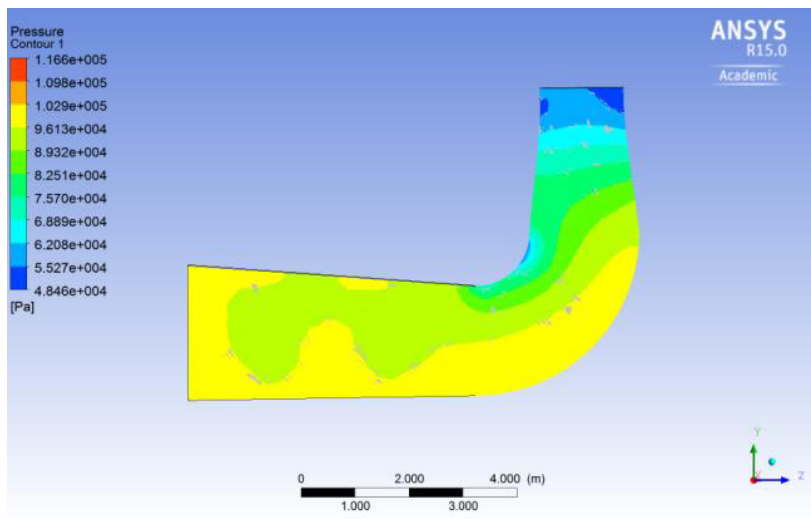

Figure11. The pressure contour for draft tube geometry. 


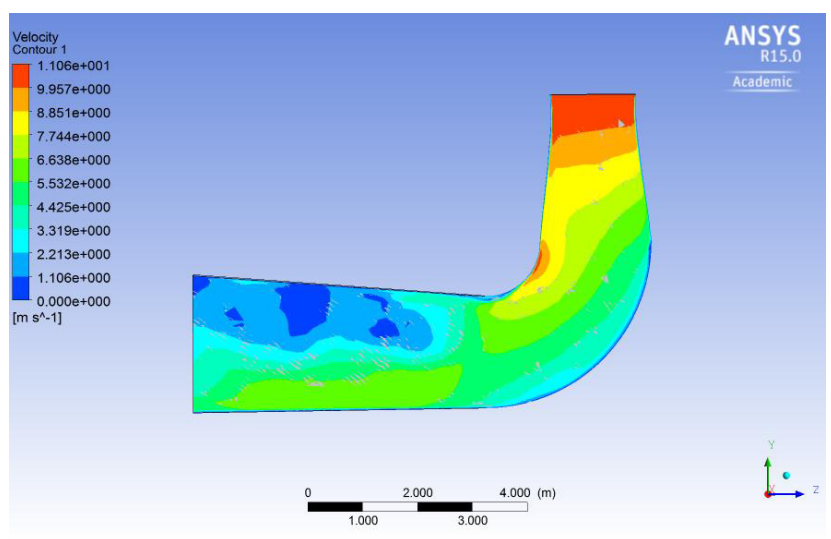

Figure12. The velocity contour for draft tube geometry

The simulation result has gives the result which estimated as the outlet velocity as $2.21 \mathrm{~m} / \mathrm{s}$. The inlet pressure has been found as $50000 \mathrm{~Pa}$ and outlet as $102900 \mathrm{~Pa}$. The head loss comes out to be $0.58 \mathrm{~m}$ and the draft tube efficiency as $86.5 \%$.

The next geometry is taken for $\boldsymbol{h}_{1} / \boldsymbol{d}_{1}$ as 1.193 , $\boldsymbol{b}_{1} / \boldsymbol{d}_{1}$ as 1.222 and $\boldsymbol{b}_{2} / \boldsymbol{d}_{1}$ as 1.666 with a mass flow rate of $20000 \mathrm{~kg} / \mathrm{sec}$. FLUENT analysis is carried out and the velocity and pressure profiles are plotted as shown in figure 13, figure 14, and figure 15 respectively as the velocity vector, pressure contour and the velocity contour.

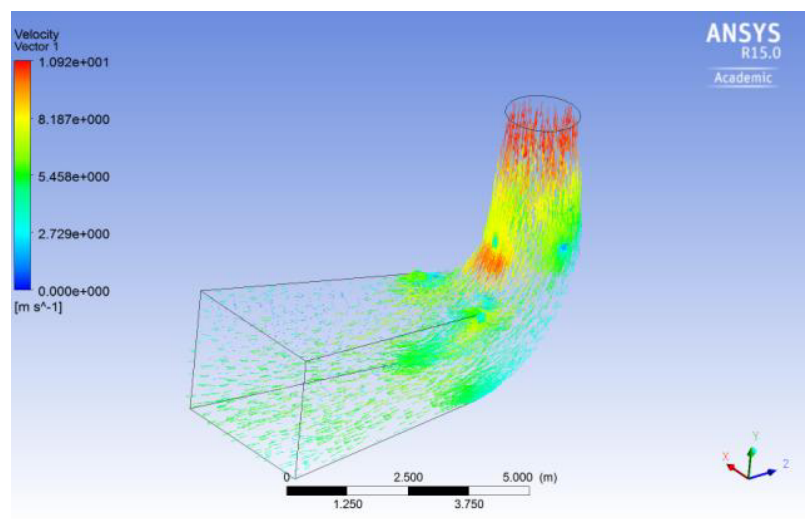

Figure 13. The velocity vector for draft tube geometry

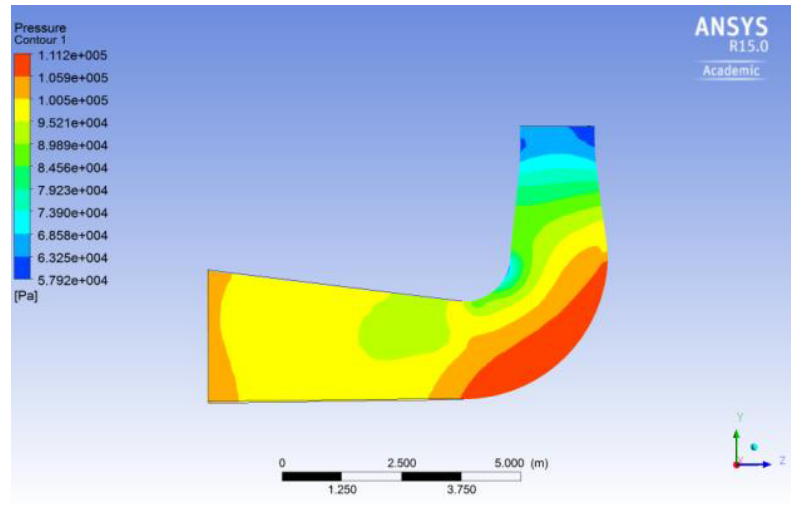

Figure14. The pressure contour for draft tube geometry

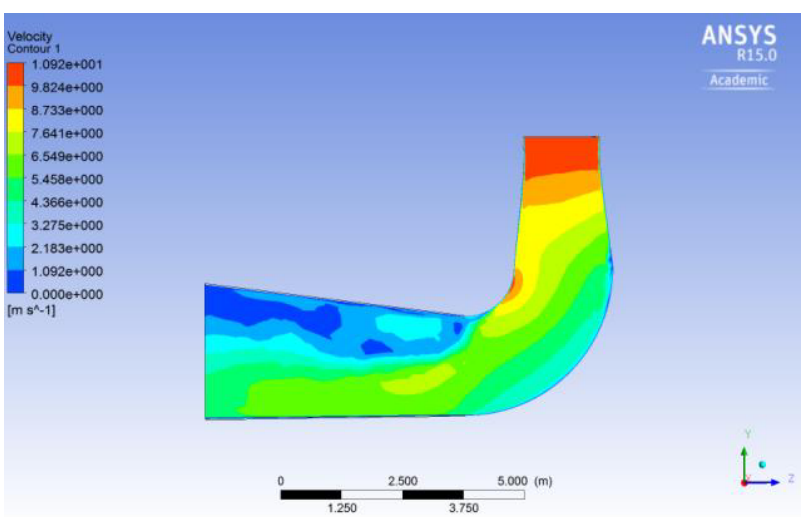

Figure 15. The velocity contour for draft tube geometry

The inlet estimated flow velocity and the pressure has been found with respect to the simulation as the out let velocity as $2.183 \mathrm{~m} / \mathrm{s}$. The inlet pressure has been found as $57920 \mathrm{~Pa}$ and outlet as $105900 \mathrm{~Pa}$. The head loss comes out to be $0.939 \mathrm{~m}$ and the draft tube efficiency as $80.47 \%$. We notice that appropriate height of the draft tube is necessary for decreasing the head loss.

\section{Conclusion}

In the present work the design optimization of the Francis turbine has been carried out in the ANSYS FLUENT simulation frame. The Geometric modelling has been established in the FLUENT CAD modelling. The tetrahedral meshing has been done for the discretization of the model, The finite volume approach has been used for the solution of the flow velocity and the pressure distribution. The draft tube efficiency has been estimated based on the simulation pressure and flow distribution. The geometric dimension of the draft tube has been varied like the length and the height of the draft tube at the exit and hence simulation has been done. The estimated velocity and pressure has used to find the draft tube efficiency. The best efficiency of the draft tube has been found as $86.5 \%$ which is quite satisfactory. The best performance is achieved with the dimension ratios as $\boldsymbol{h}_{1} / \boldsymbol{d}_{1}$ as $1.193, \boldsymbol{b}_{1} / \boldsymbol{d}_{1}$ as 1.111 and $\boldsymbol{b}_{2} / \boldsymbol{d}_{1}$ as 1.444 . It has been found from the study the efficiency of the draft tube depend on the length and the cross section area of the draft tube.

\section{References}

1. Vishnu Prasad, Ruchi khare, Abhas chincholikar, Numerical Simulation for Performance of Elbow draft tube at different geometric configurations, continuum mechanics, fluids, heat, 178-182.

2. Avellan F. Flow investigation in a Francis draft tube: the FLINDT Project. In: Proceedings of 20th IAHR symposium on hydraulic machinery and system, Charlotte, NC, US; (2000).

3. Nilsson H. Numerical investigation of turbulent flow in water turbines. Doctoral thesis, Chalmers University of Technology, Gothenbourg, Sweden; (2002). 
4. Mauri S. Numerical simulation and flow analysis of an elbow diffuser. Doctoral thesis, $E^{\prime}$ cole Politechnique Fe'de'rale de Lausanne, Lausanne, Switzerland; (2002)

5. Mohammad Hasan Shojaeefard, Ammar Mirzaei, Ali Babaei, Shape optimization of draft tubes for Agnew microhydro turbines, Energy Conversion and Management, 79: 681-689 (2014).

6. Date Ab, Date Ash, Akbarzadeh A. Investigating the potential for using a simple water reaction turbine for power production from low head hydro resources. Energy Conversion Manage;66:257-70 (2013) 